\title{
Modelling the Dynamics and Kinematics of Mechanical Systems with Multibond Graphs
}

by M. J. L. TIERNEGO and A. M. BOS

Twente University of Technology, P.O. Box 217,7500 AE Enschede, The Netherlands

ABSTRACT: A method to model mechanical systems with multibond graphs is described. The method is based on the description of the vector velocity relation of a moving point in a rotating system. This relation is incorporated in a bond graph. Since bond graphs are based on the power conserving concept, the force or momenta relations are then available too. By repeating the same bond graph structure for every point (which is of interest within a chosen coordinate frame), where each point has the same rotational velocity, a systematic way of modelling mechanical systems is achieved. It is explained how connected mechanical linkages have to be handled. Two simple examples are given.

\section{Nomenclature}

$\boldsymbol{\Omega}_{k, l}^{i}$ column matrix with the angular velocities of body $k$, with regard to body $l$, in coordinates of coordinate system $i$. The subscript $l$ is dropped when $l=0$

$\dot{\mathbf{x}}_{p, j}^{i} \quad$ column matrix with the velocity of point $p$ in coordinates of system $i$, measured with respect to the system $j$. When $j=0, j$ is dropped.

$\mathbf{F}_{p}^{i}$ column matrix with the force acting on $p$ in coordinates of system $i$

$\mathbf{A}^{j, i}$ coordinate transformation matrix to transform coordinates from system $i$ to $j$

$\mathbf{X}\left(\mathbf{x}_{p, j}^{i}\right)$ transformation matrix giving the linear velocity of point $p$ with respect to $j$, due to rotation of system $i$, in coordinates of system $i$

$\mathbf{m}_{i}$ diagonal matrix with the mass of body $i$

$\mathbf{J}_{i}$ inertia matrix of body $i$, related to the body fixed system $i$

R dissipator

C compliance

The underlined characters in the figures are equivalent to the bold face characters, representing a matrix.

\section{Introduction}

By its nature a power conserving description of a system, the bond graph method is especially practical when several physical domains have to be modelled within a system simultaneously, or when the physics of domains other than one's own specialty have to be understood. This is certainly the case in robotics where mechanic, hydraulic, pneumatic and electric systems in several combinations are present. It is beyond the scope of this paper to go into detail on the theory of bond graphs. To this purpose, other papers or books (1-3) are more suitable. Only a short introduction will be given for the understanding of this paper. Besides its contribution to the understanding of physical systems, the bond graph method can be very helpful in the preparation of equations for digital simulation. The technique used for this purpose is called causal analysis. By means of causality, implicit 
equations (e.g. dependent storage elements) can easily be detected. For the solution of these equations one may either change the model or use special numerical methods. At any rate, one is aware of their presence before simulation.

For the simulation of models obtained by bond graphs general purpose simulation programs can be used (CSMP, ACSL, TUTSIM). Three are suited for direct bond graph input [TUTSIM (4), ENPORT (5) and CAMP (6)].

In this paper a 3-D mechanical modelling technique (7) is described. This method, presented here with two simple examples, appears to be very powerful in the modelling of large mechanical systems such as robots and vehicles (8).

\section{Bond Graphs}

The bond graph is a graphical representation of the powerflow, represented by bonds, in a physical system [Fig. 1(a)]. The power, $P$, is the product of the generalized conjugated variables $e$ (effort) and $f$ (flow):

$$
P=e f \text {. }
$$

For mechanical systems the generalized variables are $F$ (force) and $v$ (velocity) or $M$ (moment) and $\Omega$ (angular velocity). The half arrow shows the direction of the powerflow if $e$ and $f$ are both positive. In 3-D mechanics a multibond graph will be used. Although this representation has been called a vector bond notation it is not a vector but merely a composition of the three bonds, corresponding to the three perpendicular axes, in one multibond. The equivalent bond graph representations are shown in Figs. 1(b). The powerflow is now defined as $P=\mathbf{e}^{T} \mathbf{f}$, where $\mathbf{e}^{T}$ is the transpose of the column vector

$$
\mathbf{e}=\left[\begin{array}{c}
e^{x} \\
e^{y} \\
e^{z}
\end{array}\right]
$$

and

$$
\mathbf{f}=\left[\begin{array}{l}
f^{x} \\
f^{y} \\
f^{z}
\end{array}\right] .
$$

Table I shows the elements used with examples in the mechanical domain. 'I hey are

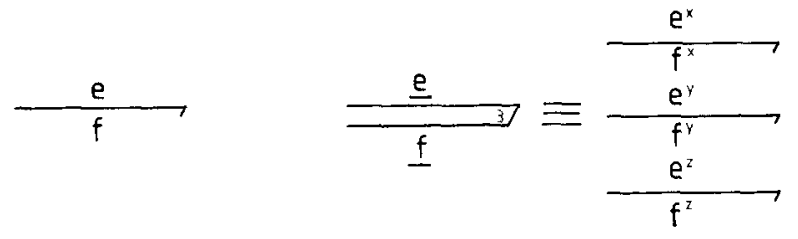

(a)

(b)

FIG. 1. (a) Single power bond. (b) Multibond. 
Table I

\begin{tabular}{|c|c|c|c|}
\hline ELEMENT & BOND GRAPH & & EQUATIONS \\
\hline So:urce & $S-\frac{F}{V}$ & & $\begin{array}{c}F(t)=\text { giver } \\
\text { or }\end{array}$ \\
\hline 1-storage & $\frac{F}{V} I$ & m & $\begin{array}{l}v(t)=\text { given } \\
v=v(0)+\frac{1}{m} \int F d t\end{array}$ \\
\hline C-storage & $\underset{V_{12}}{\longrightarrow} C$ & $\sqrt{5}$ & $F=F(0)+\frac{1}{C} \int v_{12^{d t}} d t$ \\
\hline jissipacor & 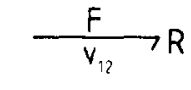 & $-F^{-v^{2}}$ & $F=f \cdot v_{12}$ \\
\hline 1-juration & $\begin{array}{c}\frac{F_{1}}{V_{1}}: \frac{F_{3}}{V_{3}} \\
F_{2} \int_{V_{2}}\end{array}$ & $\begin{array}{l}\text { parallel connection } \\
\text { in mechanical systems }\end{array}$ & $\begin{array}{l}p_{3}=p_{1}+P_{2} \\
v_{1}=v_{2}=v_{3}\end{array}$ \\
\hline o-jurction & $\frac{F_{1}}{v_{1}} \circ \frac{F_{3}}{F_{3} \mid v_{2}}$ & series connection & $\begin{array}{l}P_{3}=P_{1}+P_{2} \\
F_{1}=F_{2}=F_{3} \\
v_{3}=v_{1}+v_{2}\end{array}$ \\
\hline $\begin{array}{l}\text { (Modulated) } \\
\text { Iranoformer }\end{array}$ & $\frac{F_{1}}{v_{1}} M(t) \frac{F_{2}}{v_{2}}$ & & $\begin{array}{l}P_{1}=P_{2} \\
F_{1}=m(t) \cdot P_{2}\end{array}$ \\
\hline $\begin{array}{l}\text { (Moduleted) } \\
\text { Gyrator }\end{array}$ & $\frac{M}{\Omega} M G Y \frac{P}{\Phi}$ & $M / N_{\Omega}$ & $\begin{array}{l}P_{1}=P_{2} \\
P_{2}=r(t) \Omega \\
M=r(t) \phi\end{array}$ \\
\hline
\end{tabular}

the only ones used in the bond graphs in this paper. Although Table I shows single bonds an extension to multibonds can easily be made by replacing the bonds by multibonds and the elements by multiport elements. Two examples are shown in Fig. 2, where $\mathbf{C}^{-1}$ represents the compliance matrix and $\mathbf{m}^{-1}=m^{-1} \mathbf{I}$ is the inverse of the mass matrix and $\mathbf{I}$ is the unit matrix. For a complete and unambiguous multibond graph notation see Brecdveld (9).

One special type only used in multibond graphs has yet to be mentioned: the direct sum (9). This operation, indicated by a perpendicular stroke between separated bonds, gives the possibility of decomposition and composition of multibonds to single bonds or the other way around. An example is an electric PM motor acting on only one of the three angular velocities, as shown in Fig. 3.

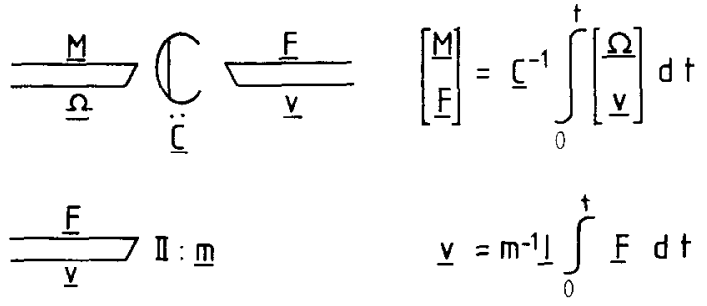

FIG. 2. Multiport elements. 


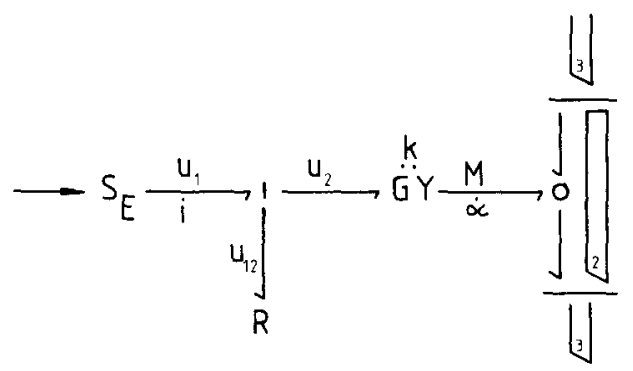

Fig. 3. Use of direct sum.

\section{Velocities in a Moving Frame}

Suppose a coordinate frame 1 (Fig. 4) rotates and translates with respect to a reference frame with origin 0 . In frame 1 a point $P$ moves with respect to 1 . The absolute velocity of $P$ is in vector notation represented by:

$$
\overline{\dot{x}}_{p}=\overline{\dot{x}}_{p, 1}+\bar{\Omega}_{1} X \overline{\dot{x}}_{p, 1}+\overline{\dot{x}}_{1,0} .
$$

This relation can be rewritten in matrix algebra form. The vector cross product has to be replaced by a matrix product, i.e.

$$
\bar{\Omega}_{1} X \bar{x}_{p, 1} \leftrightarrow \mathbf{X}\left(\mathbf{x}_{p, 1}^{1}\right) \cdot \mathbf{\Omega}_{1}^{1}
$$

with

$$
\mathbf{X}\left(\mathbf{x}_{p, 1}^{1}\right)=\left[\begin{array}{ccc}
0 & x_{p, 1}^{1 z} & -x_{p, 1}^{1 y} \\
-x_{p, 1}^{1 z} & 0 & x_{p, 1}^{1 x} \\
x_{p, 1}^{1 y} & -x_{p, 1}^{1 x} & 0
\end{array}\right]
$$

The coordinate frames in which the vectors or matrices are represented have to be indicated, which is realized by a superscript of the matrix. The matrix representation from (1) becomes

$$
\dot{\mathbf{x}}_{p, 0}^{1}=\dot{\mathbf{x}}_{p, 1}^{1}+\mathbf{X}\left(\mathbf{x}_{p, 1}^{1}\right) \boldsymbol{\Omega}_{1}^{1}+\dot{\mathbf{x}}_{1,0}^{1} .
$$

If the velocities have to be known in another coordinate frame, f.i. frame 0 , then a

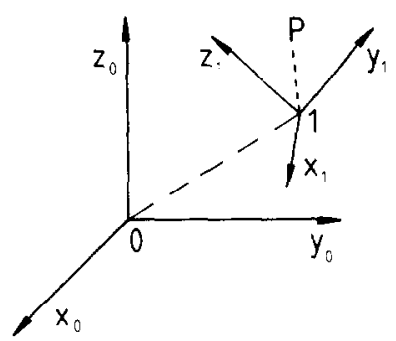

FIG. 4. Moving point in a moving frame. 


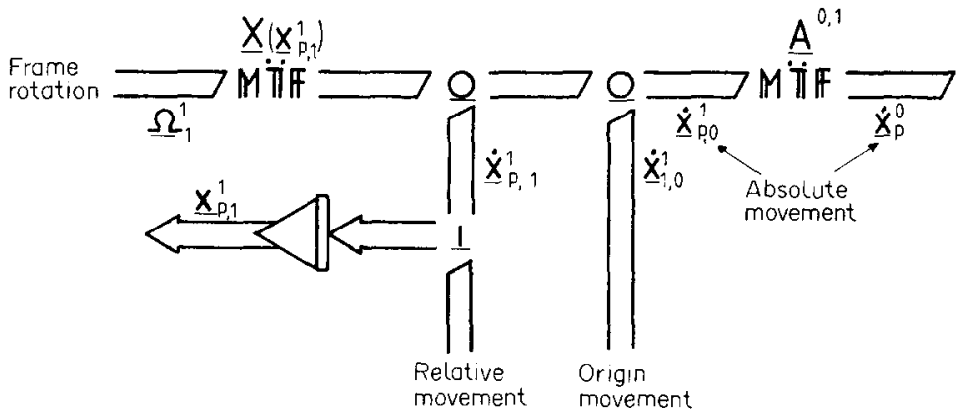

FIG. 5. Velocities in a moving frame.

coordinate transformation matrix will take care of this;

$$
\mathbf{x}_{p, 0}^{0}=\mathbf{A}^{0,1} \mathbf{x}_{p, 0}^{1}
$$

in which $\mathbf{A}^{0,1}$ is the transformation matrix from frame 1 to frame 0 . The elements of the transformation matrix have to be found by transforming the angular velocity $\Omega$ to a set of integratable angular velocities. In many applications the angles and the transformations follow from the mechanical structure, except for free bodies where no physical angles are present. For the latter case often cardan- or euler-angles or quaternions are used.

The velocity relations, (3) and (4), can be represented by the bond graph structure of Fig. 5. In this bond graph a signal block is added in order to indicate how the elements of $X(x)$ can be obtained. In a general description of a system with bond graphs there will often be a mixture of signal-flow and power-flow which, as indicated, can easily be used together. The graphical representation for power- and signal-flow are the half and full arrow respectively.

Remark. Although our starting point was the velocity of a point $P$, the corresponding force relations can easily be found from the bond graphs because the bonds represent the power flow. For example, given the modulated transformer (Fig. 6) with

$$
\dot{\mathbf{x}}_{A}^{0}=\mathbf{A}^{0,1} \dot{\mathbf{x}}_{A}^{1}
$$

and

$$
P_{1}=\mathbf{F}_{A}^{1^{T}} \dot{\mathbf{x}}_{A}^{1}=\mathbf{F}_{A}^{0^{T}} \dot{\mathbf{x}}_{A}^{0}=P_{0}
$$

it follows that

$$
\mathbf{F}_{A}^{1}=\mathbf{A}^{0,1^{T}} \mathbf{F}_{A}^{0}
$$

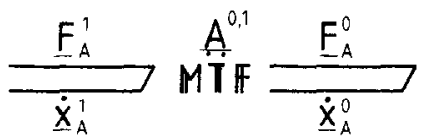

FIG. 6. Multiport modulated transformer. 
To complete the physical structure of a system the components (masses, inertias, compliances and sources) have to be added. This will be illustrated by a simple example (Example 1).

\section{Example 1 : Mass Moving in a Rotating Disc}

Consider a rotating disc with a hole (Fig. 7), in which a mass $m$ can move with friction. For simplicity only one rotation (gamma) is assumed, so the system is restricted to the two-dimensional case but can easily be extended. Two coordinate frames, $x_{0}, 0, y_{0}$ and $x_{1}, 0, y_{1}$, with coinciding origins are chosen. The $x$ axis is chosen through the hole and rotates with the disc. Gravity is assumed to be in the $-y_{0}$ direction, and the axis of rotation is perpendicular to the figure. The first step in constructing the bond graph is to represent the velocity relations. This results in the bond graph of Fig. 8, equivalent to Fig. 5, with:

$$
\mathbf{X}\left(\mathbf{x}_{m, 1}^{1}\right)=\left[\begin{array}{c}
-y_{m, 1}^{1} \\
x_{m, 1}^{1}
\end{array}\right], \quad \mathbf{A}^{0,1}=\left[\begin{array}{cc}
\cos \gamma & -\sin \gamma \\
\sin \gamma & \cos \gamma
\end{array}\right] .
$$

In the second step the elements, as described in the ideal physical model, will be added to the proper velocities. In this case they are the inertia $J$ to $\dot{\gamma}$, mass $m$ to the linear velocities in the reference frame, gravity as a force source in $-y_{0}$ direction and the force due to $\mathrm{R}$ in $x_{1}$ direction.

The constraint of the mass is modelled as a source Sf: 0 stating that the relative velocity of the mass in $y_{1}$ direction is zero. With these components the bond graph in Fig. 9 represents the complete system. Some simplifications can be made if $\mathrm{Sf}=0$ and $y_{m, 1}^{1}=0$. If necessary, the bond graph can also be represented in single bond notation (Fig. 10).

Third step: make the bond graph causal. It is beyond the scope of this paper to explain the rules for causality; refer, e.g. to Karnopp and Rosenberg $(2,3)$. Briefly, causality indicates the input and output variables for each element. This is done by assigning a perpendicular stroke to the bonds in a bond graph. This perpendicular stroke indicates the side of the bond where the effort-signal comes out. Some examples in Table II may clarify this. The causal bond graph of the example system, Fig. 7, is shown in Fig. 11.

Causality shows that there are only two independent velocities (chosen $\dot{x}_{0}$ and $\dot{y}_{0}$ ).

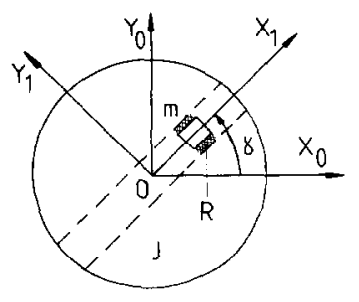

FIG. 7. Rotating disc with moving mass. 


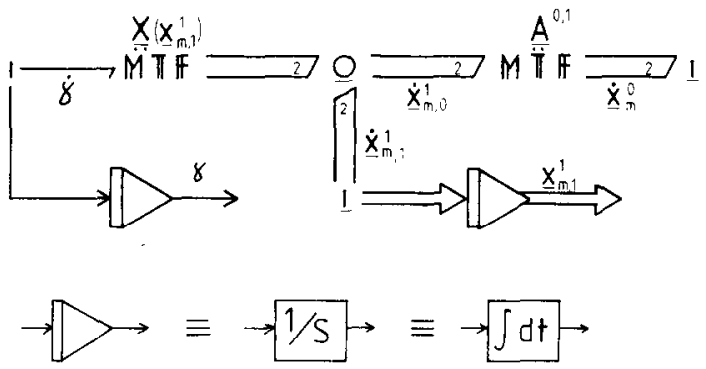

FIG. 8. Velocities of the disc.

Figure 11 also shows that a double algebraic loop exists within the MTF's, which can be eliminated by inverting $\mathbf{A}^{0,1}$. If only the equations are required then this is not necessary.

\section{$V$. Transformation of an I-element Over an $M T F$}

Sometimes it is more convenient to describe a mass in a moving coordinate frame instead of in the inertial frame. This means that the mass has to be transformed over the coordinate transformation matrix $\mathbf{A}^{0,1}$. This leads to the well known Euler equations. Because transformation over an MTF often occurs, the general formal transformation from $\mathbf{v}_{2}$ to $\mathbf{v}_{1}$ will be demonstrated for inertia $\mathbf{J}$ (Fig. 12).

By definition:

$$
\begin{aligned}
\mathbf{v}_{2} & =\mathbf{B} \mathbf{v}_{1}, \quad \mathbf{F}_{1}=\mathbf{B}^{T} \mathbf{F}_{2} \\
\mathbf{F}_{3} & =\mathbf{J}\left(\mathrm{d} \mathbf{v}_{2} / \mathrm{d} t\right)=\mathbf{J B}\left(\mathrm{d} \mathbf{v}_{1} / \mathrm{d} t\right)+\mathbf{J}(\mathrm{d} \mathbf{B} / \mathrm{d} t) \mathbf{v}_{1} \\
\mathbf{F}_{1} & =\mathbf{B}^{T} \mathbf{F}_{2}=\mathbf{B}^{T} \mathbf{F}_{3}-\mathbf{B}^{T} \mathbf{F}_{4} \\
\mathbf{F}_{1} & =\mathbf{B}^{T} \mathbf{J} \mathbf{B}\left(\mathrm{d} \mathbf{v}_{1} / \mathrm{d} t\right)+\mathbf{B}^{T} \mathbf{J}(\mathrm{d} \mathbf{B} / \mathrm{d} t) \mathbf{v}_{1}-\mathbf{B}^{T} \mathbf{F}_{4} .
\end{aligned}
$$

Only the first two terms are transformed because this results in the transformation of $\mathbf{J}$. The coefficient $\mathbf{B}^{T} \mathbf{J B}$ can be replaced by $\mathbf{J}^{\prime}$ and is called the transformed inertia. The term $B^{T} \mathbf{J}(\mathrm{d} B / \mathrm{d} t) \mathbf{v}_{1}$ is a force found by multiplying the velocity $\mathbf{v}_{1}$ by a coefficient. More generally, it is a relation between effort and flow, as occurs in

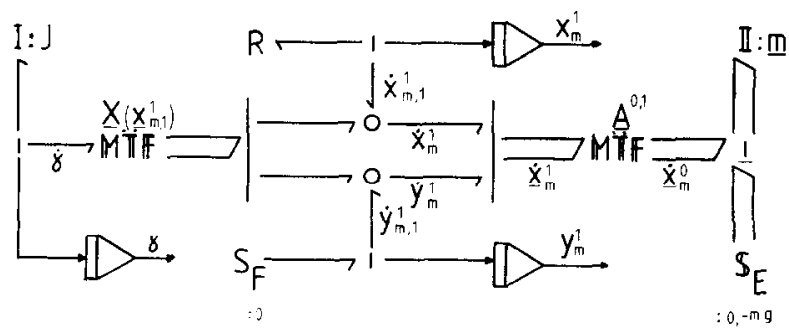

FiG. 9. Multibond graph of the disc. 


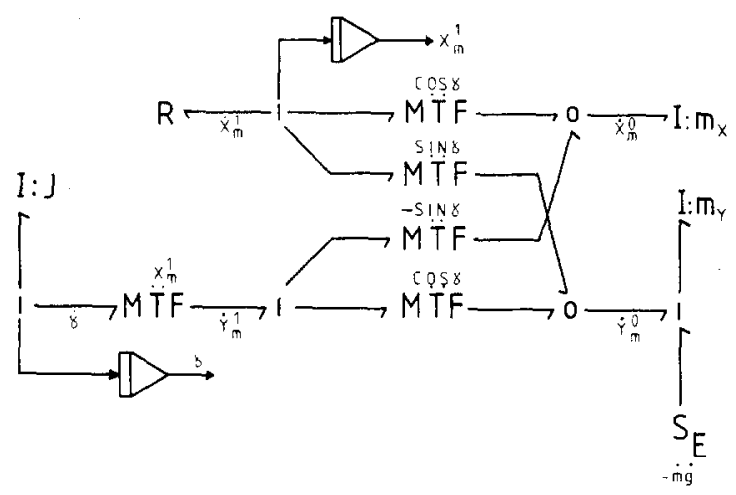

FIG. 10. Bond graph of the disc.

multiport gyrators and in resistors. This element is called a gyristor (GR), a combination of a modulated gyrator and a resistor, see Allen (10). If $\mathbf{B}$ is a coordinate transformation matrix, then $\mathbf{B}^{T}=\mathbf{B}^{-1}$ and $\mathrm{d} \mathbf{B} / \mathrm{d} t=\mathbf{B W}$, in which $\mathbf{W}$ is an antisymmetric matrix containing the rotational velocity elements. If now $\mathbf{J}$ is a mass element such that

$$
\mathbf{J}=m \mathbf{l}
$$

then

$$
\mathbf{J}^{\prime}=\mathbf{B}^{-1} m \mathbf{B}=m \mathbf{I}
$$

and

$$
\mathrm{GR}: \mathbf{B}^{-1} m \mathbf{I B}=m \mathbf{W}
$$

with $\mathbf{l}$ the unity-matrix.

The equations: $m \mathrm{l}\left(\mathrm{d}_{1} / \mathrm{d} t\right)+m \mathbf{W} \mathbf{v}_{1}=\mathbf{F}_{1}$ are called the Euler equations. The gyristor (GR) reduces in this case to a 3-port modulated gyrator, also called a Eulerian junction structure (EJS-element).

Sometimes it is necessary to transform an I-element, when a 0-junction exists between the I-element and the MTF [Fig. 13(a)]. In such a case the transformation takes two steps. First, transform the 0 -junction to the other side of the MTF [Fig.

Table II

$$
\begin{aligned}
& \frac{F}{\dot{x}} \rightarrow I: m \rightarrow \dot{x}=\dot{x}(0)+\frac{1}{m} \int_{0}^{\dagger} F . d t \text { InTEgal causality } \\
& \frac{F}{\dot{x}} \rightarrow I: m \rightarrow F=m d \dot{x} / d t \quad \text { DIFEEREnItal causality } \\
& \frac{F}{\dot{x}} \rightarrow R \rightarrow \dot{x}=F / R \\
& \frac{F}{\dot{x}} \rightarrow R \rightarrow F=R \cdot \dot{x}
\end{aligned}
$$




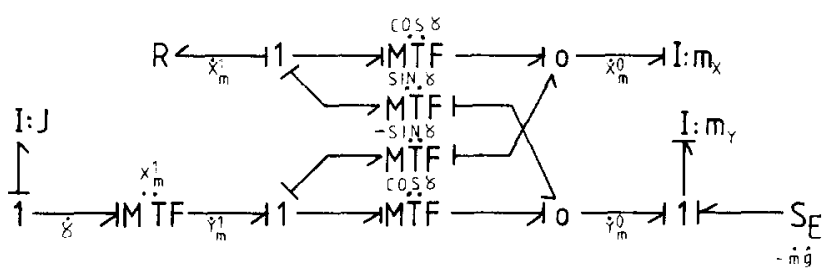

Fig. 11. Causal bond graph of the disc.

13(b)] and then the I-element can be transformed according to the rules described before. The correctness of this manipulation is shown by the following calculations. From Fig. 13(a), it follows for the velocities

$$
\mathbf{v}_{3}=\mathbf{v}_{2}+\mathbf{B} \mathbf{v}_{1}
$$

and from Fig. 13(b)

$$
\mathbf{v}_{3}=\mathbf{B}\left(\mathbf{B}^{-1} \mathbf{v}_{2}+\mathbf{v}_{1}\right) \text {. }
$$

By inspection from (9) and (10) it follows that in both cases $\mathbf{v}_{3}$ is the same.

\section{Connecting Systems Together}

Suppose a part of a system can be described by three coordinate frames $i-1, i$, $i+1$ [Fig. 14(a)]. For the origin of a system which can rotate with respect to the foregoing, the bond graph in Fig. 14(b) is valid as previously derived. The dashed line represents the frame boundary. The matrix $\mathbf{X}$ contains the position of the origin of the next frame. If the velocities of more points in the frame have to be calculated, the vertical part of the bond graph has to be repeated with a different $\mathbf{X}$-matrix. If, for example, a moving mass $m$ [Fig. 14(a)] is described within this frame the extended bond graph is shown in Fig. 15. Every other point results in such an additional structure. The complete structure will be called a subsystem or body.

A general word bond graph for the three subsystems of Fig. 14(a) is shown in Fig. 16. Using this method a complete multibond graph of any system can easily be

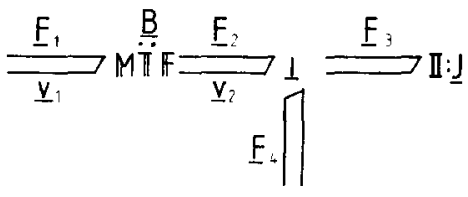

(a)

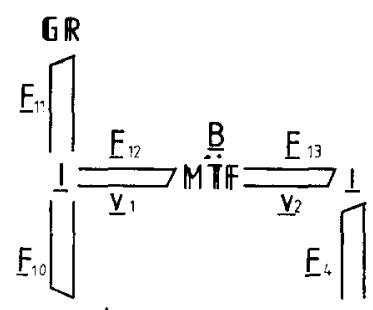

II $: \underline{\text { J' }}$

(b)

FIG. 12. Transformation of a multiport I-element over a modulated transformer. (a) Initial situation. (b) After transformation. 


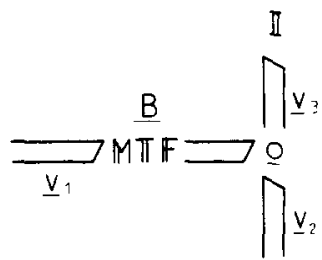

(a)

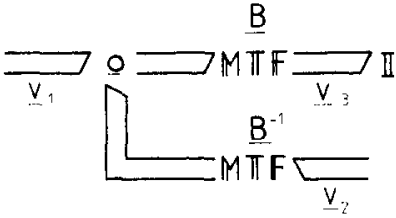

(b)

FIG. 13. First step by transformation of a multiport I element with a 0 -junction in between.

(a) Initial situation. (b) After transformation.

drawn. A graphical representation of all system interactions is then available. The next example will show the use of the method for three connected bodies.

\section{Example 2: Rate Gyro}

A rate gyro is an example of three connected bodies used as a measuring device to measure rotational velocities. In the example of Fig. 17 the velocity around the $z_{0^{-}}$ axis is the one to be measured, the input axis. The output axis is the axis $x_{1}$, where the angle $\alpha$ has to be proportional to the measured angular velocity $\dot{\gamma}$. In this example the support of the casing is supposed to be rigid. Since only two bodies move with respect to the casing, only two bodies have to be described: the gimbal (body 1) and the disc (body 2). The design is made such that their two centres of mass coincide. This appears in the bond graph as a simplification of Fig. 16 because the translational velocities disappear. In Fig. 17 an ideal physical model of a rate gyro is given.

The $\mathbf{C}-\mathbf{R}$ combination is often replaced by a torque motor and controller. The

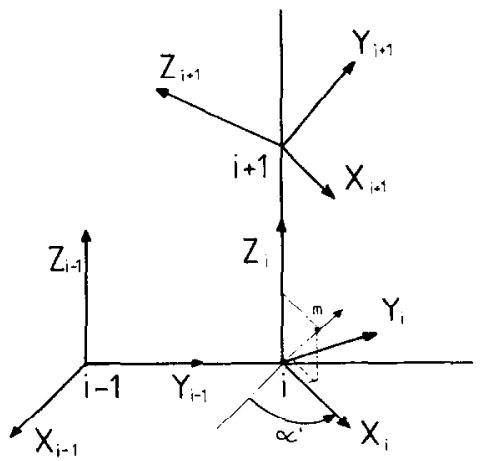

(a)

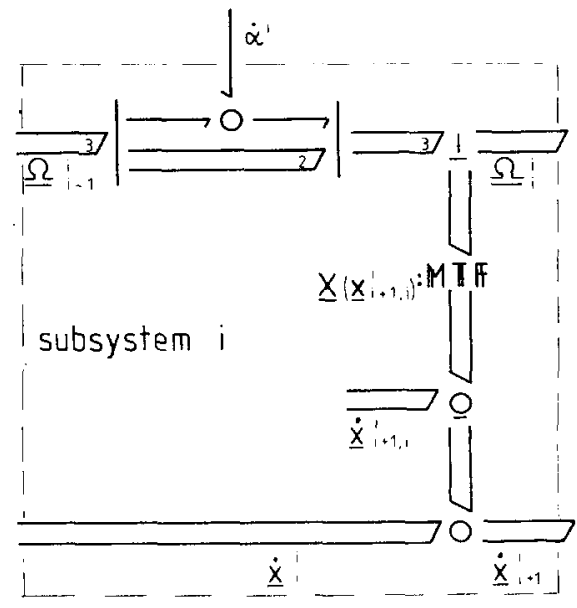

(b)

FIG. 14. (a) Two moving frames. (b) Velocities in frame $i$. 
Mechanical Systems

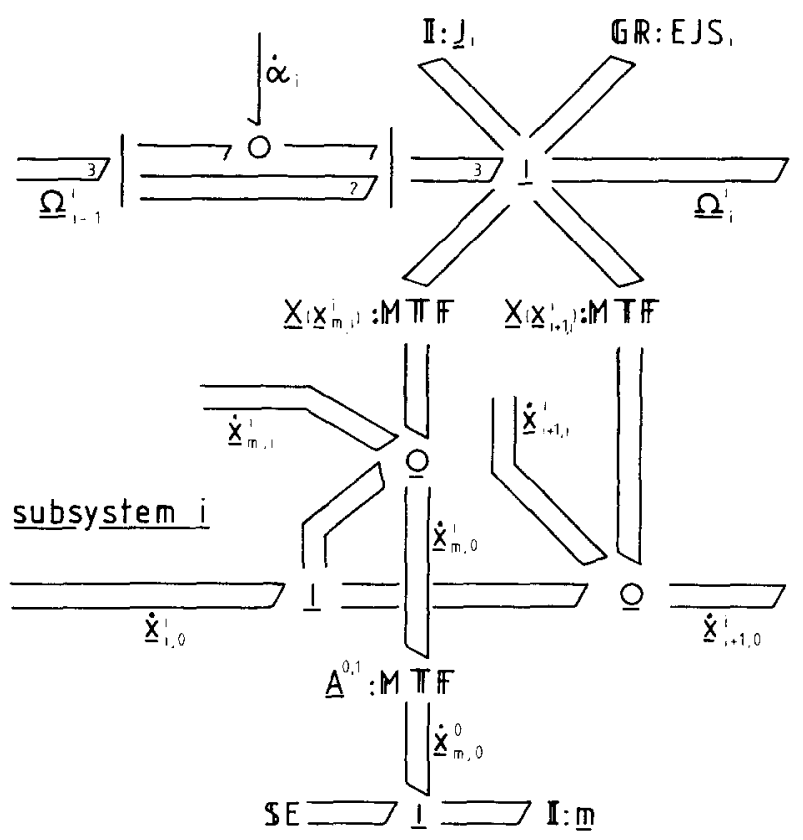

FIG. 15. Two points in the same moving frame.

word bond graph of the rate gyro is represented by that in Fig. 18, while Fig. 19 gives the multibond graph. The Sf-source at the left represents the rotational velocities sensed by the casing. The disc drive is assumed to have a constant velocity. The matrix element is given in (11):

$$
\begin{aligned}
& \mathbf{J}_{1}=\left[\begin{array}{ccc}
J_{1 x} & 0 & 0 \\
0 & J_{1 y} & 0 \\
0 & 0 & J_{1 z}
\end{array}\right], \quad \operatorname{EJS}_{1}=\left[\begin{array}{ccc}
0 & J_{1 z} \Omega_{1}^{1 z} & -J_{1 y} \Omega_{1}^{1 y} \\
-J_{1 z} \Omega_{1}^{1 z} & 0 & J_{1 x} \Omega_{1}^{1 x} \\
J_{1 y} \Omega_{1}^{1 y} & -J_{1 x} \Omega_{1}^{1 x} & 0
\end{array}\right], \\
& \mathbf{J}_{2}=\left[\begin{array}{ccc}
J & 0 & 0 \\
0 & J_{r} & 0 \\
0 & 0 & J
\end{array}\right], \quad \mathrm{EJS}_{2}=\left[\begin{array}{ccc}
0 & J \Omega_{2}^{2 z} & -J_{r} \Omega_{2}^{2 y} \\
-J \Omega_{2}^{2 z} & 0 & J \Omega_{2}^{2 x} \\
J_{r} \Omega_{2}^{2 y} & -J \Omega_{2}^{2 x} & 0
\end{array}\right]
\end{aligned}
$$

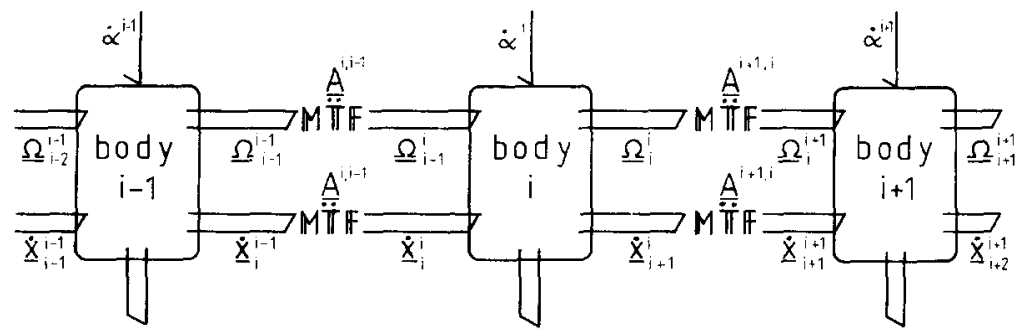

Fig. 16. Three connected moving bodies. 


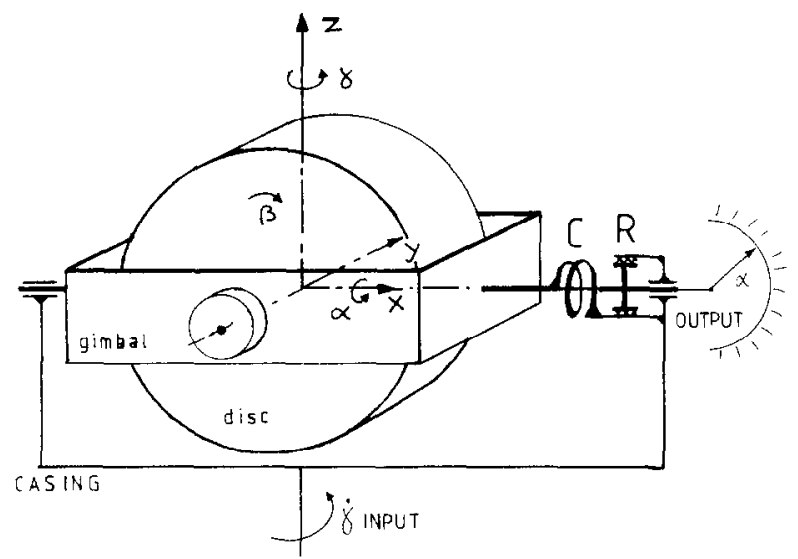

FIG. 17. Rate gyro.

As the next step a simplification is made. The components of body 2 are transformed to the components of body 1 , as this will lead to simplification of the bond graph due to the symmetry of the rotor. For the transformation the rules described in the previous section are used. Finally, the multibond graph can be drawn as a single causal bondgraph: Fig. 20. The GR-element shows up as a ring of modulated gyrators. Due to the transformation and the 0 -junction between II : $\mathbf{J}_{2}, \mathrm{GR}$ : EJS and the MTF, its elements are not the same as those of a normal EJS (11). Because of the rotor symmetry the only difference in this case is to be found in the MGY opposite of $\mathrm{I}: J_{1 y}^{\prime}$ where the component $J_{r} \beta$ is added. The dashed lines in the bond graph are drawn along the bonds which are important for the behaviour of the instrument. These are the paths along which the input signal $\dot{\gamma}$ acts on the output $\alpha$. From the causal bond graph the conclusion can be made that $J_{1 y}^{\prime}, J_{1 z}^{\prime}$ and the MGY: $J_{1 x}^{\prime} \Omega_{1}^{1 x}$ only give momenta to the casing. However $\Omega_{0}^{0 x} \quad \Omega_{0}^{0 y} \quad \Omega_{1}^{1 y}$ and $\Omega_{1}^{1 z}$ do influence the system and so the measured value $\alpha$. From the bond graph can be found that the following assumptions have to be made in order to have a proper relation between input and output: i.e.

(1) $\Omega_{0}^{0 x}=\Omega_{0}^{0 y}=0$

(2) $\hat{\beta} \gg \Omega_{1}^{1 y}$

(3) $J_{1 z}^{\prime}=\left(J_{1 z}+J\right)=J_{1 y}$

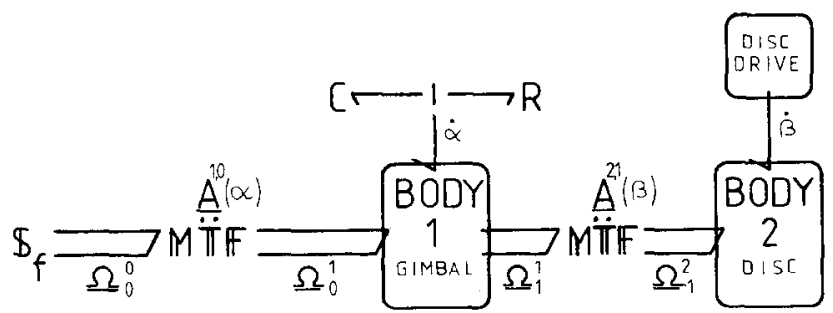

Fig. 18. Word bond graph of the rate gyro. 


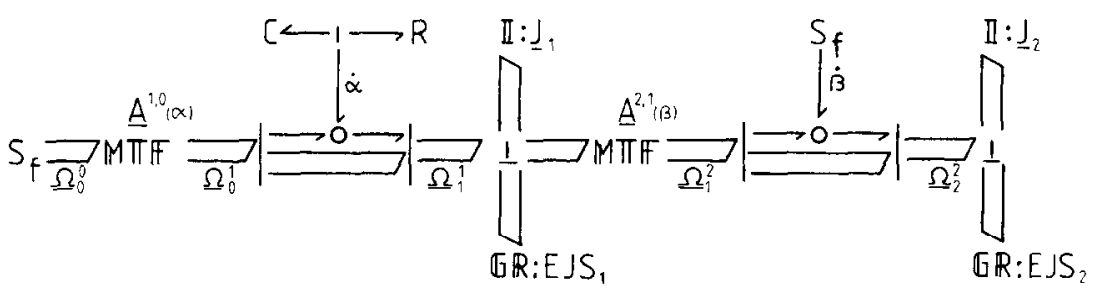

Fig. 19. Multibond graph of the rate gyro.

Then and only then the bondgraph simplifies to that of Fig. 21. From this bond graph follows Eq. (12)

$$
\alpha / \mathrm{C}+\dot{\alpha} \mathrm{R}+\ddot{\alpha} J_{1 x}=\cos \alpha J_{r} \dot{\beta} \dot{\gamma}
$$

and in the static situation the well known relation (13) is found

$$
\alpha=\dot{\gamma}\left(\cos \alpha J_{r} \beta\right) \mathrm{C} \text {. }
$$

The bond graph clarifies the conditions under which the instrument gives the expected output.

\section{Concluding Remarks}

The systematic approach by bond graphs in the modelling of mechanical systems will give in a clear way the description of a system as is shown by the two small examples. The advantage of the small examples is that the calculations and

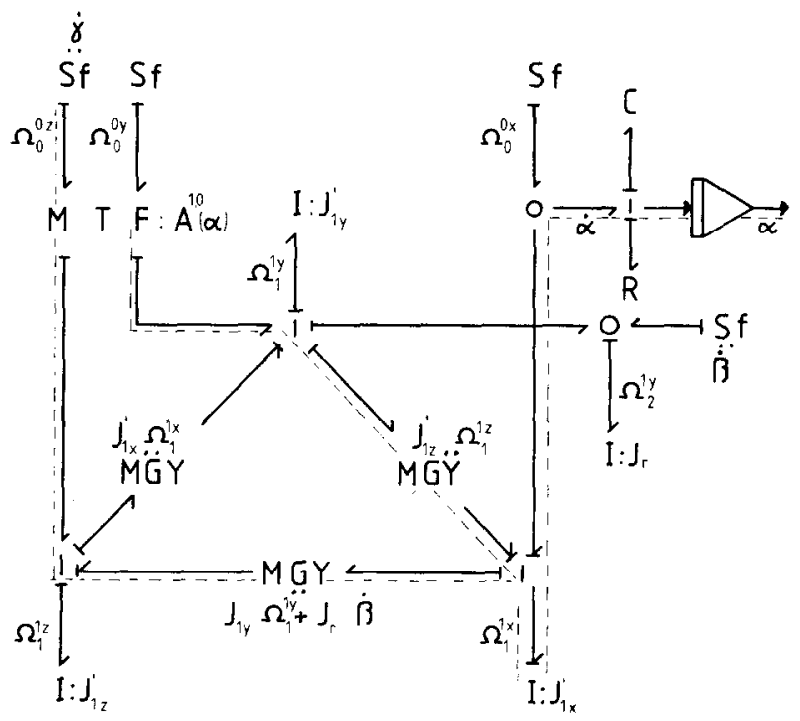

FIG. 20. Causal bond graph of the rate gyro. 


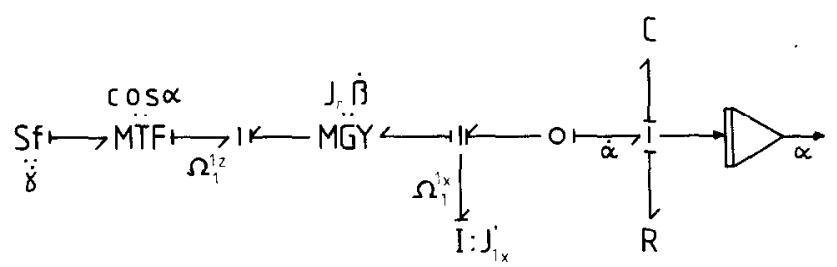

FIG. 21. Simplified bond graph of the rate gyro.

transformations still can be made by hand. But when systems are larger, as in robots, other methods as described in (8) have to be used. The basic method of describing a mechanical system, as presented in this paper, is however not only powerful for small systems but even more so for large systems. One reason is the clear and systematic presentation of the system. An advantage in the use of bond graphs is that it does not matter whether parts of the system are from other physical domains. In a companion paper, the computer assisted transformation of dependent inertias is described using formula manipulation methods (8).

\section{References}

(1) J. J. van Dixhoorn, "Bond graphs and the challenge of a unified modelling theory for physical systems". In "Progress in Modelling and Simulation" (Edited by F. E. Cellier), Academic Press, London, 1982.

(2) D. Karnopp and R. Rosenberg, "System Dynamics : A Unified Approach", Wiley, New York, 1975.

(3) R. Rosenberg and D. Karnopp, "Introduction to Physical System Dynamics", McGrawHill, New York, 1983.

(4) J. W. Meerman, "THTSIM, software for the simulation of continues dynamics on small and very small computer systems", Int. J. Modeling Simulation, Vol. 1, No. 1, 1981.

(5) R. C. Rosenberg, "A User's Guide to ENPORT-4", Wiley, New York, 1974.

(6) J. J. Granda, "A guide to using CAMP", Department of Mechanical Engineering, California State University, Sacramento, 1983.

(7) M. J. L. Tiernego, "A new systematic procedure for 3-D mechanics", Proc. 10th IMACs Congr., Montreal, 1982.

(8) A. M. Bos and M. J. L. Tiernego, "Formula manipulation in the bond graph modelling and simulation of large mechanical systems", J. Franklin Inst., Vol. 319, No. 1/2, pp. $51-65,1985$.

(9) P. C. Breedveld, "Proposition for an unambiguous vector bond graph notation", Trans. ASME J. Dynamic Syst. Measure. Control, Vol. 104, pp. 267-270, Sept. 1982.

(10) R. R. Allen, "Multiport representation of inertia properties of kinematic mechanisms", J. Franklin Inst., Vol. 308, No. 3, pp. 235-253, Sept. 1979. 